\title{
Análisis económico-financiero de los centros especiales de empleo de España
}

\author{
Economic and financial analysis of sheltered \\ employment centres in Spain
}

\section{Resumen}

Los centros especiales de empleo

(CEE) son empresas de vital

importancia para las personas con discapacidad, al facilitar su incorporación social y laboral en la sociedad. Este estudio pretende contribuir a la visibilidad académica de estas empresas, que fomentan el emprendimiento social y son socialmente responsables. El objetivo de este trabajo es el análisis económico-financiero de la estructura de los CEE de España. Para ello, se estudian sus principales indicadores financieros a través de ratios contables. Las conclusiones obtenidas señalan que apenas existen estudios económicos sobre estas empresas y que la mayoría de los CEE son pequeños, dedicados a la prestación de servicios, con forma jurídica de sociedad limitada y focalizados en Andalucía, Castilla y León, Cataluña y Madrid. En cuanto a los resultados de los ratios, de media los CEE presentan una baja rentabilidad y un alto nivel de endeudamiento a corto plazo, aunque su solvencia está garantizada.

\section{Palabras Clave}

Empleo, discapacidad, centros especiales de empleo, empleo protegido, análisis económicofinanciero, ratios contables.

\begin{abstract}
Sheltered employment centres (CEE) are important for people with disabilities as they facilitate their social and labour integration in society. This study aims to contribute to the academic visibility of these companies that are socially responsible and promote the social entrepreneurship. The objective of this paper is to analyse the economic and financial structure of these firms in Spain. To achieve this goal, their main financial indicators through accounting ratios are presented. The main conclusions are that there is almost no economic research about these companies. Most CEE are small companies limited by shares, dedicated to provide services and are located in the regions of Andalusia, Castile and León, Catalonia and Madrid. Related to the ratios, on average, the spanish CEE show a low profitability and a high level of shortterm indebtedness, although their solvency is guaranteed.
\end{abstract}

\section{Key Words}

Employment, disability, sheltered employment centres, sheltered workshop, economic-financial analysis, accounting ratios.

\author{
Vera Gelashvili \\ <veragela@ucm.es> \\ Universidad Complutense de Madrid

\section{María del Mar Camacho- Miñano} \\ <marcamacho@cunef.edu> \\ Colegio Universitario de Estudios \\ Financieros
}

\section{María Jesús Segovia-Vargas} <mjsegovia@ccee.ucm.es>

Universidad Complutense de Madrid

\begin{abstract}
Para citar:
Gelashvili, V. et al. (20I6): "Análisis económico-financiero de los centros especiales de empleo de España”. Revista Española de Discapacidad, 4 (2): 7-24.
\end{abstract}

Doi: <https://doi.org/IO. 5569/23405 IO4.04.02.0I>

Fecha de recepción: 07-I 2-20I 5 Fecha de aceptación: I 5-07-20I6 


\section{Introducción ${ }^{1}$}

El empleo tiene un papel central en el desarrollo y en la vida de todas las personas, incluso más importante para las personas con discapacidad ya que, más allá de los ingresos obtenidos por el trabajo realizado, les permiten otra serie de resultados. Para estas personas, el empleo no sólo ofrece una remuneración, sino también otros beneficios entre los que se incluyen el refuerzo de su identidad personal y el hecho de ser un medio para estructurar y ocupar su tiempo, conseguir contactos sociales, responsabilidad y participación productiva y, finalmente, dar un sentido de logro personal (Shepherd, I989). Otros investigadores consideran que dar a la población la posibilidad de mantener o conseguir un empleo es casi más beneficioso que cualquier otra intervención médica o social (Boardman et al., 2003).

Aun así, en el pasado, trabajo y discapacidad fueron incompatibles. La política básica llevada a cabo por parte de los organismos públicos fue de protección hacia las personas con discapacidad, no previendo que pudieran tener un trabajo remunerado. Pero en las últimas décadas la creciente influencia del movimiento para la personas con diversidad funcional ha desafiado esta actitud y, poco a poco, las barreras se están eliminando. El acceso de las personas con discapacidad a la formación superior, principalmente a la universidad, también ha facilitado su acceso a muchos puestos de trabajo que antes eran imposibles de conseguir.

De hecho, en varios países desarrollados se están aplicando políticas dirigidas por los gobiernos que hacen hincapié en la participación de las personas con discapacidad en el mercado de trabajo y en

I. Una versión preliminar de este artículo fue presentada en el II Congreso Internacional "Universidad y Discapacidad" en Madrid. Las autoras también agradecen las labores de orientación del editor de la revista y los comentarios de los revisores. otras actividades de la comunidad y de la vida pública (Howard, 2005). Por ejemplo, en Estados Unidos se ofrece formación profesional en talleres protegidos, un certificado especial para el trabajo asalariado, servicios previos a la formación profesional, colocaciones de trabajo en grupo y actividades de tiempo libre (Cimera et al., 2012). En otros países, como Suecia, existe un tipo de empresa de propiedad estatal que proporciona empleo protegido para personas con discapacidades severas (denominada Samhall) que, además de proporcionar empleo para los trabajadores con discapacidad, les ofrece también rehabilitación fuera del Samhall (Skedinger y Widerstedt, 2003).

En España, entre las estrategias realizadas a favor del empleo de personas con discapacidad (véase entre otros, el Real Decreto 290/2004, sobre la regulación de los enclaves laborales como medidas de fomento de empleo de las personas con discapacidad), el Gobierno ha incentivado la creación de Centros Especiales de Empleo (CEE). Éstos tienen su origen en la Ley I3/I982, de 7 de abril, de Integración Social de los Minusválidos (LISMI), que tenía como principales aspectos básicos la integración sociolaboral de las personas con discapacidad.

La definición actual de los CEE se recoge en el Real Decreto Legislativo I/20I3, de 29 de noviembre, que define a los CEE como "las empresas cuyo objetivo principal es el de realizar una actividad productiva de bienes o de servicios, participando regularmente en las operaciones del mercado, y tienen como finalidad el asegurar un empleo remunerado para las personas con discapacidad; a la vez que son un medio de inclusión del mayor número de estas personas en el régimen de empleo ordinario". Además de los servicios de ajuste personal y social de los CEE, éstos permiten ayudar a superar las barreras, obstáculos o dificultades que los trabajadores con discapacidad de estos centros tienen en el proceso de incorporación a un puesto de trabajo, así como la permanencia y progresión en el mismo (Real Decreto 469/2006, de 2 I de abril).

Los CEE han de tener en su plantilla al menos un $70 \%$ de personas con discapacidad igual o superior a un $33 \%$. Además, estas empresas 
cuentan con ciertos beneficios económicos (como subvenciones en la cuota de la seguridad social o para adaptación al puesto de trabajo) y pueden crearse desde la iniciativa pública, privada o mixta, de manera indistinta, ya sea con o sin ánimo de lucro (Giménez et al., 2012). Pueden tener la forma jurídica que se desee pero es indispensable su calificación e inscripción en el Registro de Centros del Servicio Público de Empleo Estatal (SPEE) o en el correspondiente de las Administraciones Autonómicas.

Esta consideración se consigue mediante el cumplimiento de todos los requisitos exigidos por la ley, además de la presentación de una memoria explicativa de la situación y actividades del centro (Camacho-Miñano y Pérez, 20I2).

Son muchas las ventajas que ofrecen los CEE para las personas con discapacidad, entre otras, la labor social de crear empleo para este colectivo con más garantías que el empleo ordinario. Además en los CEE la calidad del empleo es bastante satisfactoria (Gómez et al., 20IO) y durante la crisis han desempeñado un papel de "refugio de empleo", evitando que las personas con discapacidad pierdan el contacto con el mercado de trabajo (Rodríguez, 20I2). Analizando la literatura existente sobre CEE se destaca su importancia para las personas con discapacidad, favoreciendo su complicada integración social y laboral (De Lorenzo, 2004; Cueto et al., 2008; Calderón y Calderón, 2OI 2; Redondo y Martin, 20I4). Los estudios realizados por varios investigadores en España han revisado la evolución de los CEE en los últimos años (Laloma, 2007; Cueto et al., 2008; Rodríguez et al., 2009; Jordán de Urríes y Verdugo, 20Io; Camacho-Miñano y Pérez, 20I2; Gelashvili et al., 20I 5) y los resultados obtenidos muestran su importante crecimiento durante las dos últimas décadas. Teniendo en cuenta esta evolución, se puede considerar que los CEE son empresas rentables. Sin embargo, los CEE, al igual que el resto de empresas, se enfrentan al problema de su viabilidad económico-financiera (Gelashvili et al., 20I 5), aspecto que apenas ha sido tratado y, en ocasiones de manera parcial, en la literatura previa (Redondo, 20I3; Redondo y Martin, 20I4; Gelashvili et al., 20I 5); aunque ninguno de ellos analiza los principales ratios contables utilizando la muestra de todos los CEE de España. Por lo tanto, el objetivo de este estudio es el análisis de la estructura económicofinanciera de los CEE de España a través de los ratios contables más importantes.

La principal contribución de este estudio es que se constata, una vez analizada la literatura previa, que apenas existen estudios empíricos sobre estas empresas desde un enfoque económico. La mayoría de los CEE en España son empresas pequeñas, dedicadas a la prestación de servicios, con forma jurídica de sociedad limitada y situados en las comunidades autónomas de Andalucía, Cataluña, Castilla y León y Madrid. Los resultados del análisis económico-financiero de los CEE muestran que son empresas solventes, especialmente a corto plazo, presentan una baja rentabilidad y una elevada deuda acumulada. Todo ello garantiza su viabilidad futura en el mercado, apostando por el empleo de las personas con discapacidad.

\section{Antecedentes. La importancia de los CEE en la sociedad}

El sector de la economía social agrupa actualmente a más de 42.900 empresas en España, lo que representa el Io \% del PIB en facturación y casi dos millones y medio de empleos (CEPES, 2OI $5^{2}$ ). Son empresas competitivas, generadoras de empleo, que están comprometidas con las personas y tienen por finalidad construir una sociedad más equitativa, solidaria e integradora en el plano social y económico. De hecho, se trata de un sector plural y diverso, que aglutina cooperativas, sociedades laborales, mutualidades, empresas de inserción y cofradías de pescadores, entre otras. También los CEE tienen una gran importancia en el marco de la economía social.

Las personas con discapacidad pueden encontrar un puesto de trabajo, apoyo educativo y facilidades para su incorporación social y laboral a través de los CEE. De hecho, la formación y el empleo son

2. Para más información véase: http://www.cepes.es 
los dos ámbitos en los que se ha desarrollado, con una mayor intensidad, el movimiento asociativo de las personas con discapacidad (Calvo, 2004). Un estudio realizado por KPMG destaca que la tasa de empleo de personas con discapacidad en España se sitúa en valores inferiores al $30 \%$, por debajo de la que se registra en otros países avanzados como Suecia, Alemania o Reino Unido (KPMG, 20I3). Debe considerarse que la dificultad de las personas con discapacidad a la hora de obtener y conservar un empleo puede ser superable con la adopción de políticas adecuadas, tendentes a la integración de este colectivo preferentemente en el mercado de trabajo ordinario, o en su defecto en el mercado protegido, con el objetivo claro y el convencimiento de que el empleo es el paso fundamental para la integración social de las personas con discapacidad (Alcaide, 2005).

En los últimos años, diversas iniciativas parlamentarias y gubernamentales pro discapacidad han favorecido el desarrollo de programas de rehabilitación específicos, han apoyado la inserción laboral adaptándose al entorno social y han facilitado la coordinación entre las entidades colaboradoras externas tales como administraciones locales, autonómicas, ONG, etc. (Barea y Monzón, 2008; Huete y Díaz Velázquez, 20I I). Asimismo, las empresas públicas o privadas que empleen a 50 o más trabajadores están obligadas a que al menos $2 \%$ de éstos sean trabajadores con discapacidad (Real Decreto 364/2005, de 8 de abril). Pero en comparación con otras medidas, los CEE son la principal política de integración laboral de las personas con discapacidad en España. De hecho, la "Estrategia Global de Acción para el Empleo de Personas con Discapacidad 2008-20I2" los sigue reconociendo como tales, aunque se trate también de reforzar su función de puente hacia el empleo ordinario. Sin embargo, hay que admitir que podrían servir como empleo estable para los trabajadores que, debido a su grado de discapacidad y dependencia, tengan dificultades para entrar en el mercado ordinario, y como camino de vuelta cuando un trabajador no se adapte al empleo ordinario (Cueto et al., 2008).

No obstante, la plena integración de las personas con discapacidad en el entorno social todavía es un problema (Calderón y Calderón, 20I2) y el apoyo que, tanto el preparador laboral como el supervisor natural o el compañero de trabajo más inmediato, proporcionan al trabajador con discapacidad resulta imprescindible en las fases iniciales de su inserción y también para el mantenimiento del empleo. Además, el apoyo de la familia es muy importante, ya que generalmente contribuye a reforzar y ejercitar las habilidades que el trabajador debe mejorar (Alomar y Cabré, 2005). Por eso, la Estrategia Española sobre Discapacidad 20I22020 tiene como propósito facilitar a las personas con discapacidad todos sus derechos, que están centrados fundamentalmente en la educación, integración laboral, pobreza y exclusión social.

En resumen, podemos afirmar que la efectividad de los CEE como instrumento de integración laboral es válida, sobre todo para aquellas personas con discapacidad que presentan más dificultades de inserción laboral, contribuyendo a reducir sus índices de paro, así como su inactividad y su integración en la sociedad (Cueto et al., 2008; Rodríguez et al., 2009; Calderón y Calderón, 20I2). Una de las tareas pendientes de los CEE es aumentar su percepción pública como organizaciones modernas, eficientes y que contribuyen de manera significativa y directa a la riqueza nacional (KPMG, 20I3). En la última década se ha avanzado positivamente en la integración de las personas con discapacidad en la sociedad española y para que los principios de solidaridad, cooperación y compromiso cívico sean los motores que rijan nuestra sociedad con el fin último de darles visibilidad social y política.

En España muchos trabajos han estudiado el comportamiento económico financiero de diferentes sectores empresariales (entre otros, Fernández y García, I991; Fernández et al., I996; De Andrés, 2000; Carmona et al. 2013), aunque hay pocos estudios empíricos sobre la situación económico-financiera de los CEE (Redondo, 2013; Redondo y Martin, 2014; Gelashvili et al., 20I 5). Teniendo en cuenta todo lo expuesto anteriormente, consideramos interesante analizar la situación económico-financiera de los CEE, empresas que tienen un valor para la sociedad y para la economía del país. En el siguiente epígrafe, analizaremos de manera descriptiva la situación reciente de este tipo de empresas. 


\section{Análisis descriptivo de los CEE de toda España}

A finales del año 2013 había en España I.834 CEE. Ya que la regulación y organización de este tipo de empresas son competencia autonómica, salvo en el País Vasco, Melilla y Ceuta que dependen del Ministerio de Empleo, la información se encuentra muy desagregada. Sólo hay una comunidad autónoma, Madrid, que publica información detallada sobre sus CEE con acceso gratuito. Otras comunidades autónomas, como Aragón y Galicia, tienen la información sobre los CEE existentes accesible a través de internet, aunque es incompleta y, por tanto, sesgada. En el resto de las comunidades no es posible el acceso online a la información relativa a sus CEE. También existen diversas confederaciones de CEE (tales como Aceca, Feacem, Conacee, Fecetc o Fecepas) pero no hay ninguna que aglutine a todos ellos a nivel nacional. Por este motivo, se hace complicado un estudio detallado y global de este sector.

A través del Servicio de Seguimiento de la Gestión y Apoyo al Empleo de Personas con Discapacidad adscrito a la Secretaría de Estado para el Empleo (SEPE), dependiente del Ministerio de Empleo y Seguridad Social, se pudo acceder al nombre de todos los CEE existentes en nuestro país. Después del tratamiento y limpieza de los datos, se buscó su información económica-financiera en la base de datos de empresas SABI.

El primer criterio utilizado para el análisis de los CEE es su localización geográfica. La mayoría de estas empresas están ubicadas en cuatro comunidades autónomas: Andalucía, Castilla y León, Cataluña y Madrid. Como se observa en el gráfico I, en la comunidad de Andalucía hay 308 CEE, lo que representa un I6,7\% del total nacional. Curiosamente, según la Encuesta de Población Activa, en junio del año 2013 esta comunidad autónoma era la que aglutinaba el mayor porcentaje de desempleo nacional $(35,7 \%)$. Castilla y León está en segundo lugar con 222 centros ( I 2, I \% del total), Cataluña ocupa el tercer puesto con 2 I 2 CEE (I I, $5 \%$ del total) y la Comunidad de Madrid cuenta con el I० \% del total de estas empresas. Lógicamente, Ceuta y Melilla son los territorios que, dada su extensión en tamaño, tienen menos centros.

\section{Grafico 1. CEE por comunidades autónomas (2013)}

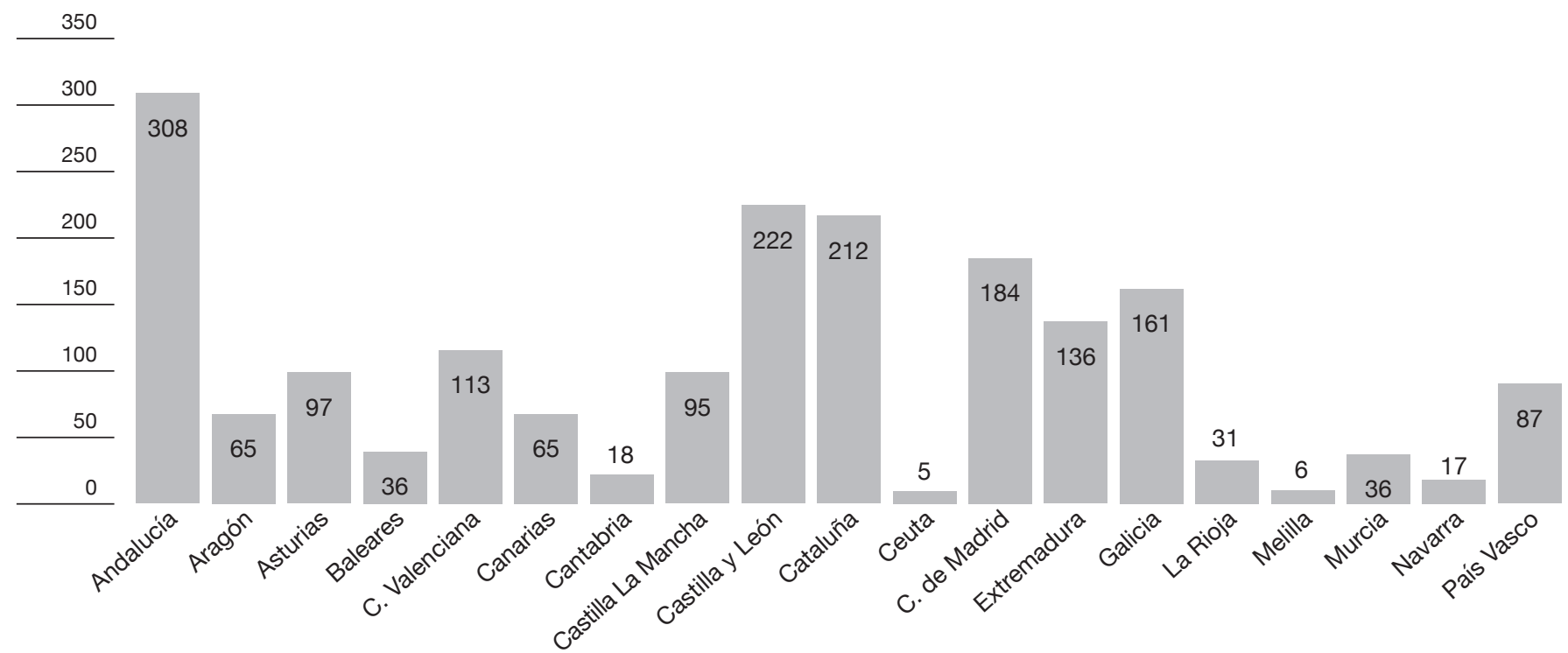

Fuente: elaboración propia a través de la documentación facilitada por el Servicio Público de Empleo Estatal. 
Si comparamos esta distribución con el número global de empresas existentes en España, hay que destacar que Andalucía (alrededor de 470.000 empresas, $17,4 \%$ del total nacional) y Castilla León (alrededor de I 62.000 empresas, $6 \%$ del total nacional) no son las comunidades autónomas que mayor número de empresas tienen. También es interesante subrayar el elevado porcentaje de CEE en Galicia, Extremadura y Asturias, comunidades de pequeña extensión territorial y con poco tejido empresarial global. De estas cifras y su comparativa a nivel nacional se desprende que la existencia de CEE depende de la sensibilidad política de unas comunidades frente a otras. De hecho, al estar los CEE regulados por cada comunidad autónoma, unas legislaciones favorecen más su creación frente a otras, con ayudas específicas y planes de apoyo especiales. Madrid y Cataluña son comunidades emprendedoras y concentradoras de empleo a nivel nacional pero hay otras que, sin tener esa vocación, son emprendedoras y concentradoras de empleo para las personas con discapacidad.

El segundo criterio de análisis es la forma jurídica que tienen estas empresas, que podría ser una proxy de su tamaño. Es decir, las sociedades anónimas, al requerir una cifra mínima de capital social de más de 60.000 euros, son de mayor tamaño que las sociedades limitadas, en las que se exige por ley un capital mínimo de 3.000 euros. En el gráfico 2 se observa que existe una gran disparidad en cuanto a la forma jurídica de estas empresas, desde asociaciones a cooperativas, pasando por congregaciones e instituciones religiosas y corporaciones locales. Sin embargo, la mayoría de estos centros son empresas con formas jurídicas tradicionales. El $65 \%$ de los CEE son sociedades de responsabilidad limitada, frente al I $6 \%$ que tienen forma jurídica de asociaciones y el ı० \% de ellos que son sociedades anónimas.

\section{Grafico 2. Forma jurídica de los CEE. Porcentajes}

Otros tipos no definidos en el resto de claves

Congregaciones e instituciones religiosas

Organismos públicos

Corporaciones locales

Sociedades civiles, con o sin personalidad jurídica

Asociaciones

Sociedades corporativas

Sociedades de resposabilidad limitada

Sociedades anónimas

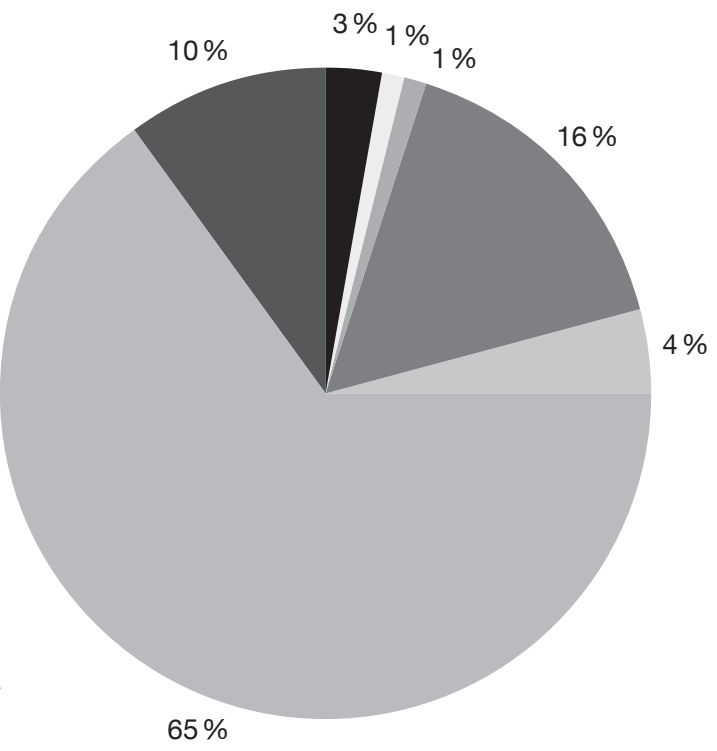

$65 \%$

Fuente: elaboración propia a través de la documentación facilitada por el Servicio Público de Empleo Estatal. 
La idea que subyace del anterior gráfico es que los CEE no son centros ocupacionales en ningún caso, sino empresas. De hecho el artículo 9 del Real Decreto 2273/I985, de 4 de diciembre, por el que se aprueba el Reglamento de los CEE establece literalmente que se "vendrán obligados a realizar una gestión sujeta a las mismas normas y requisitos que los que afectan a cualquier empresa del sector a que pertenezcan”.

En cuanto al criterio de la actividad que desarrollan, los CEE de España pertenecen a diversos sectores, como se observa en el gráfico 3. En primer lugar, señalar que se dedican a actividades muy diversas, desde el manipulado e industria hasta publicidad, marketing y artes gráficas. Sin embargo, hemos encontrado muchas empresas que no están adscritas a ninguna actividad y también CEE que realizan múltiples actividades (35\% del total). Destacan las empresas de actividades de limpieza ( $6,8 \%$ ), manipulados ( $16,7 \%$ ) y transporte $(5,4 \%)$. Las dos primeras actividades, limpieza y manipulados, requieren de tareas rutinarias en su mayoría, que suelen ser realizadas de manera muy competente por los trabajadores con discapacidad.

\section{Grafico 3. CEE según la actividad que desarrollan (2013)}

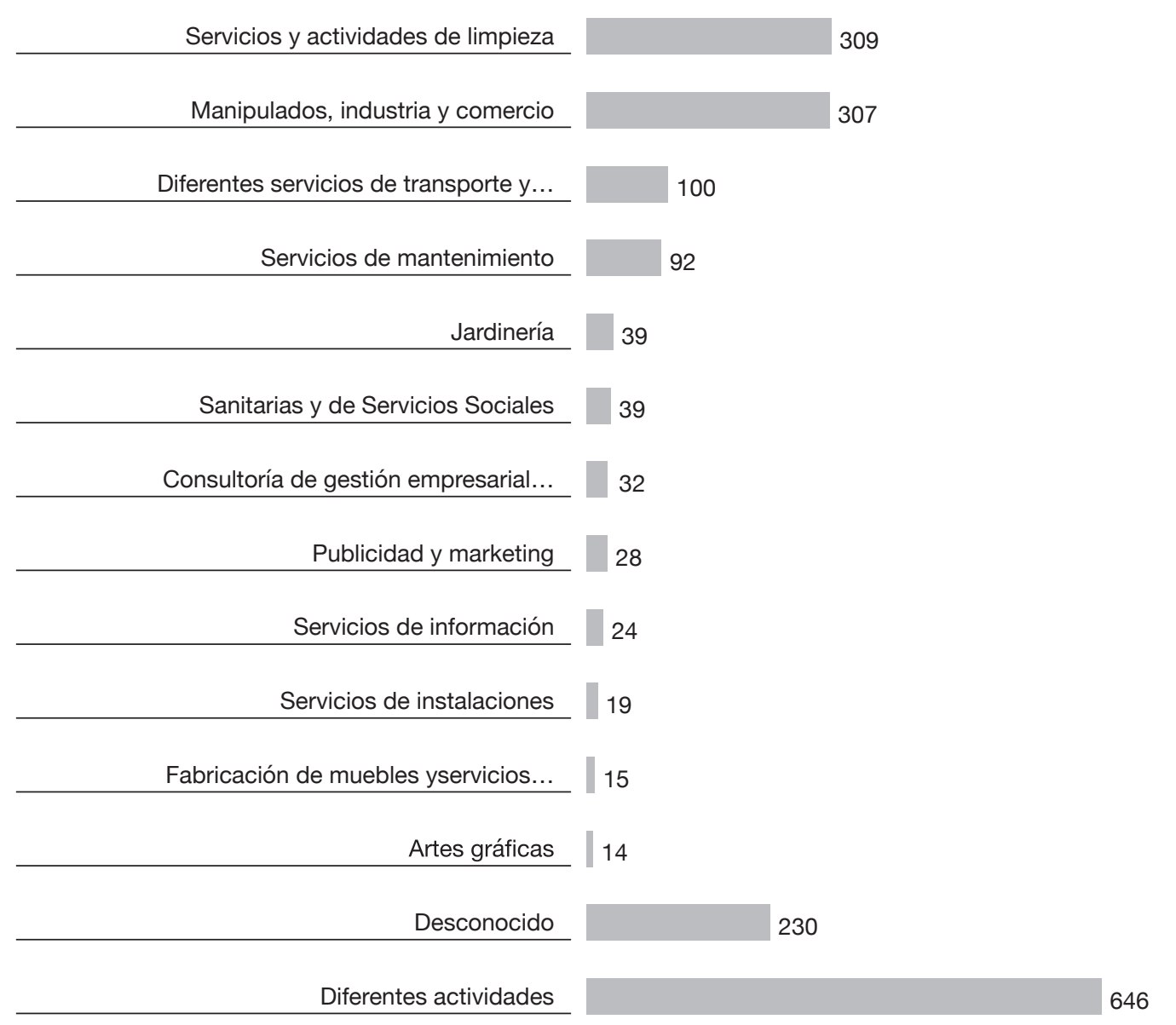

Fuente: elaboración propia a través de Servicio Público de Empleo Estatal. 
Si agrupamos estas actividades por sectores, el principal es el de servicios. Este resultado está en línea a nivel estatal con la información del Instituto Nacional de Estadística (INE), que recoge que el $70 \%$ del tejido empresarial español está formado por empresas de servicios. Concretamente las actividades de limpieza y mantenimiento de edificios son las actividades empresariales con una estabilidad asegurada en el mercado, debido a que la demanda es estable e incluso creciente, ya que en muchos hogares este servicio se hace prácticamente imprescindible. Esto está motivado por el incremento de la capacidad adquisitiva media en los hogares donde los dos miembros trabajan y la incorporación plena de la mujer al mercado laboral. Específicamente, la actividad de limpieza es necesaria para el desempeño diario de las empresas e instalaciones. De hecho, según el informe especial de DBK, "Grupos Multiservicios”, el $30 \%$ de las franquicias también agrupan estas actividades. Según datos del INE en el año 20I3 las actividades industriales de limpieza (código 8 2 del CNAE$2009^{3}$ ) agrupan a más de 24.000 empresas dedicadas a estas actividades, ocupando a más de 462.000 trabajadores. Es de destacar que casi el $80 \%$ de estos trabajadores son mujeres que, comparado con los hombres, acumulan mayores tasas de desempleo. Sin embargo, este sector tiene una competencia muy agresiva, con remuneraciones bajas -muy ajustadas al salario mínimo interprofesional- y con escasa fidelidad por parte de algunos clientes.

\section{Muestra y metodología del estudio}

\subsection{Muestra}

A través del número de identificación fiscal de las empresas hemos podido acceder a las cuentas anuales de los CEE, utilizando la base de

3. CNAE-2009 son las siglas de clasificación nacional de actividades económicas, revisado el año 2009. datos SABI. En el momento de realizar nuestro estudio, el último año disponible de las cuentas anuales fue 2013. Sin embargo, no fue posible obtener los datos del balance de situación y la cuenta de resultados de todos los CEE de España. La muestra de la investigación al final se limitó a $530 \mathrm{CEE}$, lo que supone el $29 \%$ de la muestra total ${ }^{4}$.

La media de antigüedad de las empresas de la muestra es de I I años, es decir que tienen experiencia en el mercado. En cuanto a las actividades que desarrollan los CEE de la muestra final, los datos han sido recodificados siguiendo el sistema de clasificación de las actividades NACE rev.2. En el grafico 3 se muestran los CEE según SEPE. Sin embargo, al reducirse la muestra dicha clasificación estaba muy sesgada. Por este motivo hemos utilizado los códigos por actividades incluidos en SABI. Según esta información, el $26 \%$ de estas empresas desarrollan sus actividades en el área de actividades administrativas y servicios auxiliares (esta sección incluye las actividades de limpieza), el $25 \%$ están operando en la industria (alimentaria, textil, extractivo, manufacturera) y el i $8 \%$ realizan actividades de comercio al por mayor y al por menor, reparación de vehículos, transporte y almacenamiento. Por último, el Io \% de las empresas se dedican a prestar servicios de informacion y comunicación, actividades profesionales, científicas y técnicas. Los restantes realizan actividades diversas.

Según la forma jurídica de las empresas de la muestra final, el $90 \%$ son sociedades de responsabilidad limitada, el $9 \%$ de estas empresas son sociedades anónimas y el I \% restante presentan diferentes formas jurídicas.

En el grafico 4 se presenta la distribución por localización de la muestra final.

\footnotetext{
4. Hay que tener en cuenta que algunos de los centros no están obligados a presentar sus cuentas en el Registro Mercantil. Esto hace que muchas empresas presten poca atención en la elaboración de la informacion contable.
} 


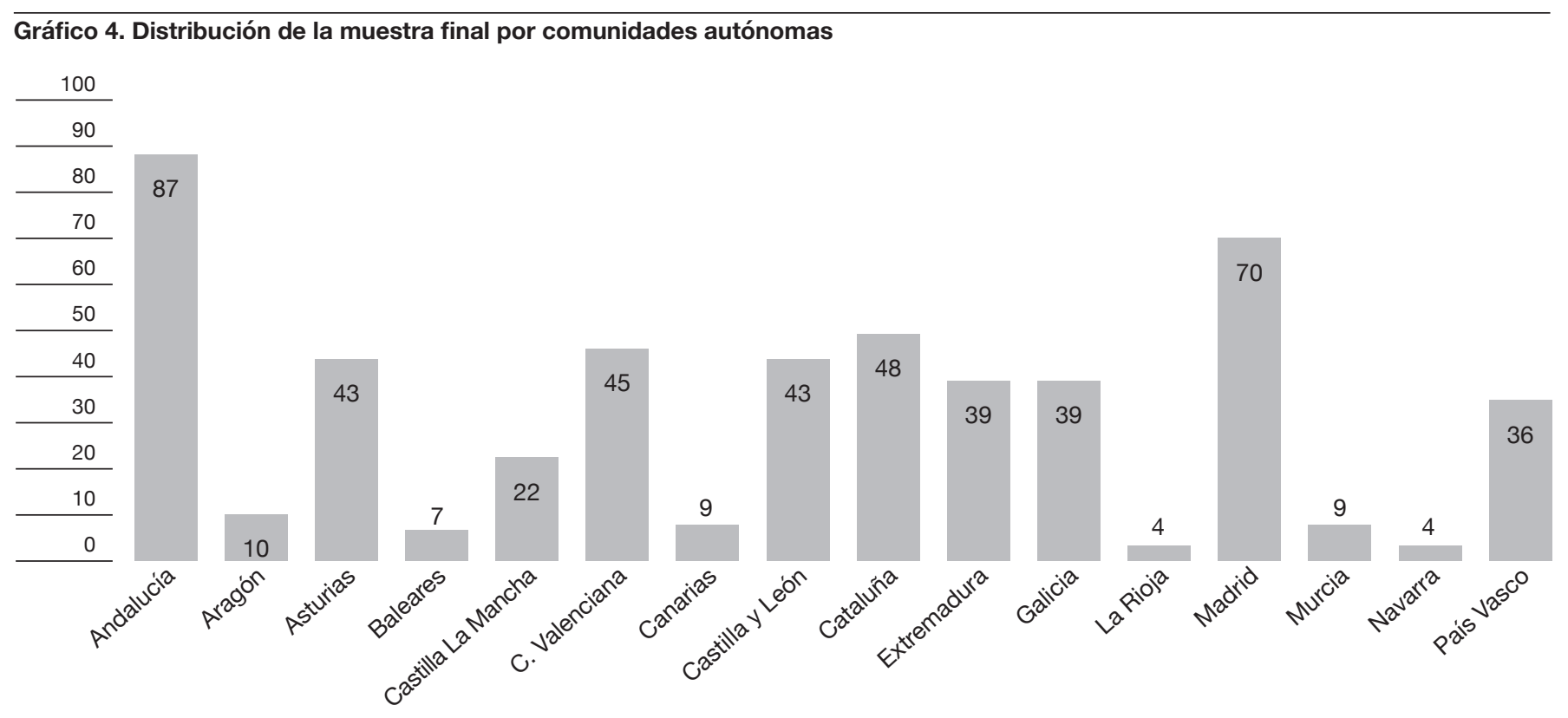

Fuente: elaboración propia a través de la base de datos SABI.

Según el gráfico 4 , las comunidades autónomas que mayor número de CEE tienen en la muestra final son Andalucía, la comunidad de Madrid, Cataluña y la comunidad Valenciana. Las ciudades de Ceuta y Melilla no disponían de datos financieros para 2013, por eso están excluidas de la muestra final.

\subsection{Los ratios contables utilizados}

Las variables utilizadas son aquellas que sirven para analizar la situación económico-financiera actual de los CEE. Normalmente, para el análisis económico-financiero de una empresa se utilizan ratios contables o razones/proporciones entre dos variables económicas. Un estudio realizado por Carmona et al. (20I3) utiliza los ratios para el diagnóstico económico-financiero de las empresas cooperativas. Dada las similitudes de gestión entre CEE y las cooperativas, se han usado aquellos ratios que mejor analizan si una empresa es rentable, si está endeudada y si es solvente. A continuación se presentan cada una de estas variables (véase tabla I):
I. Los ratios de rentabilidad determinan si la empresa genera los suficientes recursos en función de los medios económicos o financieros de los que dispone. Existen diferentes tipos de rentabilidad empresarial. Las más utilizadas son la rentabilidad económica o ROA, que mide la eficiencia de la empresa a través de sus inversiones y la rentabilidad financiera o ROE, que trata de medir la capacidad de remuneración de la empresa a favor de sus accionistas. Cuanto mayor sea el ratio de rentabilidad, más eficiente será la empresa y mejor desarrollará su actividad principal, objeto social de la misma. Hemos incluido en el análisis otros ratios de rentabilidad como RENI, REN2, $\mathrm{REN}_{3}$ y $\mathrm{REN}_{4}$ que miden la rentabilidad comparando los resultados antes de impuestos con las inversiones realizadas por los propietarios, con los recursos propios, con los recursos ajenos y las ventas. Todos ellos se recogen en porcentajes.

2. Los ratios de endeudamiento o de estructura financiera determinan si la 
empresa tiene demasiado nivel de deudas y analizan la proporción del patrimonio neto en relación a sus deudas a corto o a largo plazo; es decir, la relación entre la financiación externa y la autofinanciación que posee la empresa. END representa la totalidad de la deuda acumulada por la empresa, $\mathrm{EF}_{\mathrm{I}}$ y EF2 analizan la deuda de la empresa a corto y largo plazo, y EF3 y EF4 son los ratios que miden el coste de la financiación ajena y los gastos financieros en las ventas.
3. Los ratios de solvencia a corto/largo plazo analizan si una empresa puede hacer frente o no a sus deudas a corto/largo plazo. Se dice que una empresa es solvente a corto/ largo plazo si puede pagar sus deudas a corto/largo plazo. Por lo tanto, los ratios SOLV_CPI y SOLV_CP2 miden la capacidad de la empresa para pagar sus deudas a corto plazo y las ratios SOLV_LP3 y SOLV_LP4 analizan la solvencia de la empresa a través de recursos generados y miden la solvencia de la empresa globalmente.

Tabla 1. Ratios contables analizados

\begin{tabular}{|c|c|c|}
\hline \multicolumn{3}{|c|}{ Ratios de rentabilidad } \\
\hline ROA\% & (Resultado Explotación/Total Activo)*100 & $\mathrm{RE} / \mathrm{TA}$ \\
\hline ROE\% & (Resultado del Ejercicio /Fondos Propios) ${ }^{\star} 100$ & RE/FP \\
\hline REN1\% & (Resultado del Ejercicio antes de impuestos/Total Activo)*100 & $\mathrm{REAI} / \mathrm{TA}$ \\
\hline REN2\% & (Resultado del Ejercicio antes de impuestos /Fondos Propios)*100 & $\mathrm{REAI} / \mathrm{FP}$ \\
\hline REN3\% & (Resultado del Ejercicio antes de impuestos /Pasivo Total)*100 & REAI /PT \\
\hline REN4\% & (Resultado del Ejercicio/Importe neto Cifra de Ventas)*100 & RE/INCV \\
\hline \multicolumn{3}{|c|}{ Ratios de endeudamiento } \\
\hline END & Pasivo Total/Patrimonio Neto & PT/PN \\
\hline EF1 & Pasivo no Corriente/Fondos Propios & $\mathrm{PNC/FP}$ \\
\hline EF2 & Pasivo Corriente/Fondos Propios & $\mathrm{PC} / \mathrm{FP}$ \\
\hline EF3 & Gastos Financieros/Pasivo Total & GF/PT \\
\hline EF4 & Gastos Financieros/Importe Neto Cifra de Ventas & GF/INCV \\
\hline \multicolumn{3}{|c|}{ Ratios de solvencia } \\
\hline SOLV_CP 1 & Activo corriente/Pasivo Corriente & $\mathrm{AC} / \mathrm{PC}$ \\
\hline SOLV_CP 2 & (Activo corriente - Existencias)/Pasivo Corriente & $(A C-E) / P C$ \\
\hline SOLV_LP 3 & $\begin{array}{l}\text { (Resultado del Ejercicio+ Impuestos sobre sociedades+ Inmovilizado } \\
\text { material)/Pasivo Total }\end{array}$ & $(\mathrm{RE}+\mathrm{ISS}+\mathrm{IM}) / \mathrm{PT}$ \\
\hline SOLV_LP4 & Patrimonio neto/Pasivo Total & PN/PT \\
\hline
\end{tabular}

Fuente: elaboración propia a través de la base de datos SABI. 


\section{Resultados}

\subsection{Ratios de rentabilidad}

En la tabla 2 se recogen los resultados de los ratios de rentabilidad de los CEE de la muestra. La rentabilidad económica de los CEE es del I, $4 \%$ lo que supone que son empresas con baja rentabilidad. De cada roo euros de inversión en activo, se genera I,4 euros de resultado de explotación; es decir, generan I,4 euros por la realización de su actividad principal. Si comparamos la rentabilidad de los CEE con empresas similares, como pueden ser las cooperativas, podemos ver que no hay mucha diferencia, incluso los CEE resultan más rentables en todos los aspectos menos en la rentabilidad de las ventas, donde los CEE durante 20I3 han tenido un resultado negativo $(-2,2 \%)$. Asimismo se puede observar que las inversiones realizadas por los propietarios en CEE son más altas $(20,52 \%)$ que en las cooperativas (9\%). Para profundizar en los resultados se ha efectuado un análisis por cuartiles, ya que dicho análisis permite dividir los CEE en cuatro partes iguales y analizar cada una de ellas. La tabla 2 muestra que los CEE situados en el primer cuartil tienen, en media, rentabilidad económica (ROA) negativa $(-23,79 \%)$, mientras que los cuartiles 2, 3 y 4 presentan una rentabilidad económica positiva. También es llamativa la cifra de rentabilidad de los CEE del cuarto cuartil $(23,75 \%)$, lo que supone que hay centros que tienen una rentabilidad muy alta, mientras que otros luchan para sobrevivir en el mercado con rentabilidades prácticamente nulas. En cuanto a la rentabilidad financiera (ROE) de los CEE, es del I $6,4 \%$ lo que supone que de cada roo euros que invierten los accionistas, se recuperan I6,4 euros en el ejercicio. Si comparamos ROA y ROE, la media del ROA es significativamente más baja que de la ROE, eso implica que los CEE tienen más capacidad de remuneración a favor de sus accionistas.

Si comparamos los diferentes tipos de rentabilidades, la más elevada es la que compara los resultados con los fondos propios de la empresa seguida de la que los compara con el endeudamiento de la empresa. El ratio $\mathrm{REN}_{4}$ que compara el resultado del ejercicio con el importe neto cifra de ventas presenta la cifra es más baja comparando con otros ratios de rentabilidad. De hecho, la rentabilidad obtenida por las ventas no ha sido positiva para los CEE en el período de estudio. Quizás el impacto de la crisis económica ha sido el causante de una disminución de las ventas, en línea con lo que plantean Hernández y Millán (2015).

Sin embargo, los ratios de rentabilidad más elevados son también los que tienen mayor desviación típica, ROE y REN2, lo que supone que hay un mayor grado de dispersión de las observaciones respecto a la media. Analizando los cuartiles de los ratios de rentabilidad, se observa que el $50 \%$ de los CEE son empresas rentables y llegan a una rentabilidad satisfactoria $\left(Q_{3} ; Q_{4}\right)$. El $25 \%$ de los CEE de la muestra final presentan una rentabilidad negativa (QI) y el otro $25 \%$ presenta bajos resultados economico-financieros $\left(\mathrm{Q}_{2}\right)$.

Tabla 2. Estadísticos descriptivos de los ratios de rentabilidad. CEE 2013
\begin{tabular}{|l|c|c|c|c|c|c|c|c|}
\hline Variable & Mínimo & Máximo & Q1 & Q2 & Q3 & Q4 & Media & Desv. típica \\
\hline ROA\% & $-224,25$ & 308,67 & $-23,79$ & 0,79 & 5,15 & 23,75 & 1,48 & 26,76 \\
\hline ROE\% & $-1378,81$ & 5372,34 & $-61,44$ & 2,36 & 12,36 & 112,44 & 16,4 & 252,3 \\
\hline REN1\% & $-224,25$ & 280,02 & $-25,64$ & $-0,003$ & 4,23 & 23,24 & 0,46 & 26,88 \\
\hline REN2\% & $-1378,81$ & 7163,12 & $-73,44$ & 2,34 & 13,88 & 139,48 & 20,52 & 329,9 \\
\hline REN3\% & $-564,02$ & 433,57 & $-39,89$ & 0,00 & 9,16 & 77,14 & 11,58 & 65,68 \\
\hline REN4\% & $-315,26$ & 368,42 & $-38,33$ & $-0,19$ & 4,60 & 25,11 & $-2,20$ & 39,51 \\
\hline
\end{tabular}

Fuente: elaboración propia a través de la base de datos SABI 


\subsection{Los ratios de endeudamiento o de estructura financiera}

En la tabla 3 se resumen los principales ratios que miden el endeudamiento de los CEE. El valor óptimo de este ratio END se sitúa entre 0,4 y o,6. Cuando el valor de ratio es superior a 0,6 indica que la empresa tiene un endeudamiento elevado. Los CEE están fuertemente endeudados, es decir, de cada roo euros de financiación interna, de media, soportan 377 euros de financiación externa. Sin embargo, hay una gran dispersión. Esta variación supone un riesgo de gran endeudamiento para algunos CEE, lo que les podría llevar a una posible descapitalización empresarial, además de elevados costes financieros.

En cuanto al vencimiento de las deudas, se observa que el endeudamiento a largo plazo (EFI) es muy inferior al de corto plazo (EF2). Es decir, que los CEE tienen, como media, 0,37 euros de deudas a largo plazo por cada euro de fondos propios, mientras que alcanzan 3,4 euros de deudas con vencimiento a corto plazo por cada euro de fondos propios. Asimismo el ratio que mide los costes de financiación ajena $\left(\mathrm{EF}_{3}\right)$ presenta un $2 \%$ del total de los pasivos, lo que implica que su coste no es elevado, a pesar de estar fuertemente endeudados. El ratio $\mathrm{EF}_{4}$, que compara los gastos financieros con las ventas, tiene un valor inferior a 0,04 . Esto significa que los gastos financieros sobre ventas no son excesivos. En líneas generales, estas empresas están siendo financiadas a bajo coste, quizás debido al impacto de las ayudas públicas o la posibilidad de endeudamiento sin coste a través de organismos públicos como el Instituto de Crédito Oficial (ICO).

En cuando a los cuartiles podemos decir que las empresas que están situadas en el cuarto cuartil tienen un endeudamiento bastante alto, mientras que centros que están en el primer cuartil presentan un endeudamiento negativo. De allí podemos decir que $25 \%$ de los CEE de la muestra final están fuertemente endeudados. En cuanto a los ratios $\mathrm{EF}_{3}$ y $\mathrm{EF}_{4}$ vemos que la diferencia entre los cuatro cuartiles es escasa, eso significa que estos dos ratios tienen un impacto similar en los resultados financieros de los CEE.

\begin{tabular}{|c|c|c|c|c|c|c|c|c|}
\hline Variable & Mínimo & Máximo & Q1 & Q2 & Q3 & Q4 & Media & Desv. típica \\
\hline END & $-101,34$ & 914,27 & $-4,04$ & 0,57 & 1,58 & 17,00 & 3,77 & 40,99 \\
\hline EF1 & $-68,66$ & 31,97 & $-1,16$ & 0,03 & 0,23 & 2,81 & 0,37 & 4,50 \\
\hline EF2 & $-49,13$ & 914,27 & $-2,49$ & 0,37 & 1,10 & 14,65 & 3,40 & 40,43 \\
\hline EF3 & 0,00 & 0,13 & 0,00 & 0,005 & 0,01 & 0,06 & 0,02 & 0,02 \\
\hline EF4 & 0,00 & 1,66 & 0,00 & 0,002 & 0,01 & 0,09 & 0,03 & 0,12 \\
\hline
\end{tabular}

Fuente: elaboración propia a través de la base de datos SABI. 


\subsection{Ratios de solvencia a corto/largo plazo}

En la tabla 4 podemos observar los principales estadísticos descriptivos de los ratios de solvencia a corto y largo plazo. Para los ratios de solvencia, el valor ideal es un valor igual o superior a I,5. En general, los datos demuestran que los CEE son empresas solventes, especialmente en el corto plazo. Los ratios SOLV_CPI y SOLV_CP2 tienen una media casi igual a 3 . Esto indica que con los recursos disponibles a corto plazo pueden hacer frente a sus deudas con un vencimiento inferior a I 2 meses, aunque su desviación típica también es alta. El ratio que menos valor presenta con respecto a la solvencia de los CEE es SOLV_LP 3 con una media de o,80, que compara los recursos generados antes de impuestos con la deuda total de la empresa. Para el funcionamiento de las empresas en su día a día, lo importante es que tengan unos ratios de solvencia a corto plazo superiores a los ratios de solvencia a largo. Esto les otorga una mayor capacidad a la hora de desarrollar su objeto social.

Analizando el ratio de solvencia SOLV_LP4, también llamado de garantía, que mide la solvencia global de los CEE a largo plazo, vemos que hay una gran dispersión aunque, de media, la garantía ofrecida a sus acreedores es elevada.

\section{Si analizamos la solvencia de los CEE} por cuartiles, vemos que un $25 \%$ de las empresas presentan una solvencia muy alta, independientemente del plazo, es decir, tanto en el largo como en el corto plazo. Las empresas que se sitúan en los cuartiles 2 y 3 tienen una solvencia a corto plazo más alta que la solvencia a largo plazo, pero siguen siendo empresas solventes. En caso del primer cuartil, QI, se observa que la solvencia a largo plazo es negativa para las empresas que están en este cuartil, mientras que solvencia a corto plazo es positiva, aunque presentando un valor bastante bajo.

\section{Conclusiones}

El objetivo de este estudio es el análisis económico-financiero de los CEE en nuestro país. En primer lugar, hemos constatado que existen muy pocos estudios previos sobre la viabilidad económico-financiera de este tipo de empresas. En segundo lugar, los

\begin{tabular}{|c|c|c|c|c|c|c|c|c|}
\hline Variable & Mínimo & Máximo & Q1 & Q2 & Q3 & Q4 & Media & Desv. típica \\
\hline SOLV_CP 1 & 0,01 & 80,31 & 0,61 & 1,22 & 2,03 & 7,65 & 2,87 & 5,74 \\
\hline SOLV_CP 2 & 0,01 & 80,24 & 0,46 & 1,09 & 1,85 & 7,43 & 2,71 & 5,73 \\
\hline SOLV_LP 3 & $-5,36$ & 29,19 & $-0,18$ & 0,32 & 0,74 & 2,33 & 0,8 & 1,74 \\
\hline SOLV_LP4 & $-0,85$ & 128,55 & $-0,09$ & 0,42 & 1,23 & 7,44 & 2,25 & 7,77 \\
\hline
\end{tabular}

Fuente: elaboración propia a través de la base de datos SABI. 
análisis muestran que los CEE están situados principalmente en cuatro comunidades autónomas, son mayoritariamente sociedades limitadas y están centrados en la prestación de servicios. Como hemos constatado realizando este estudio, existen dificultades para acceder a los datos de estas empresas. Salvo en la Comunidad de Madrid, la información pública recogida en internet está sesgada o no existe. Esta falta de transparencia de la información conlleva una falta de visibilidad de esta realidad social y, en ocasiones, justifica la poca sensibilidad hacia el empleo de las personas con discapacidad.

Analizando los datos financieros de los CEE disponibles a finales del año 2013 se observa que sus rentabilidades medias son bajas, ya que sus activos no generan suficiente rendimiento en la realización de su actividad principal, si bien son rentables para sus accionistas. Por tanto, cumplen con el mandato del Real Decreto Legislativo I/20I3 de ser empresas cuyo objetivo principal no es maximizar sus beneficios sino realizar un trabajo productivo, participando regularmente en las operaciones del mercado. En cuanto al endeudamiento, se observan unas elevadas tasas de deuda a corto plazo, por término medio, lo que pone en cuestión la viabilidad presente de algunos CEE, aunque son empresas solventes. La pregunta que subyace a continuación es la siguiente: ¿qué factores podrían condicionar la solvencia de estas empresas? Una posible futura línea de investigación deriva hacia un análisis más profundo de la situación económico-financiera de los CEE, añadiendo variables que podrían ser explicativas de estos resultados. Una de estas variables podría ser la cuantía de las ayudas públicas, o la localización de la empresa en una determinada región.

Las perspectivas de empleo de una persona con discapacidad no están simplemente determinadas por su grado de discapacidad, ya que las políticas de empleo protegido, de rehabilitación y apoyo para su integración en el mercado de trabajo ordinario son claves para su normalización laboral y social. Dentro del empleo para personas con discapacidad, los CEE son una buena estrategia, tanto empresarial como social, para cumplir con la responsabilidad empresarial hacia la sociedad. Estas empresas, recogidas dentro del epígrafe de economía social, constituyen una salvaguarda para la verdadera normalización e integración de personas con discapacidad. Nadie está exento de un accidente grave o una enfermedad, por lo que los organismos públicos deben potenciar el rol que estas empresas juegan en la sociedad actual. Es más, en un contexto de crisis como el actual, el emprendimiento se ha convertido en una salida a la misma. El emprendimiento social se traduce en la creación de empresas en las que los fines sociales son la prioridad y el beneficio empresarial no es más que el resultado del trabajo bien hecho, y no la maximización del beneficio económico. Para promover la colaboración institucional público-privada para el desarrollo de iniciativas emprendedoras de carácter social, la Asociación Española de Contabilidad y Administracion de empresas (AECA) ${ }^{5}$ ha constituido un grupo de trabajo sobre empresa social. Esta iniciativa tiene una firme vocación de dar visibilidad a las empresas sociales, identificando claramente sus necesidades y tratando de comprender mejor este modelo de organización.

Finalmente, para mejorar la situación existente hay que remover las barreras socioculturales, legales y físicas, tanto arquitectónicas como de comunicación, que aún dificultan el acceso al empleo de las personas con discapacidad. Habría que realizar campañas informativas dirigidas a empresarios, profesores y a otros agentes sociales sobre materias tales como: la diversidad de los tipos de discapacidad, las capacidades laborales potenciales de las personas con alguna discapacidad, la existencia de los CEE, su legislación y viabilidad como parte del emprendimiento social, etc... Además de esto hay que poner en marcha campañas de motivación dirigidas a las personas con discapacidad para que se encaminen hacia la vida laboral activa: informando sobre recursos

5. http://www.compromisoempresarial.com/tercersector/ emprendedores-sociales-2/20I 4/o3/aeca-constituye-un-grupode-trabajo-sobre-empresa-social/ 
formativos, recursos para acceder al mercado de trabajo, o sobre cómo inscribirse como demandantes de empleo, sensibilizando de manera específica a las pequeñas y medianas empresas y a los trabajadores sin discapacidad sobre las potencialidades de las personas con discapacidad y sobre los beneficios que para todos supone compartir actividad con ellos, en línea con el informe de "Estrategia Global de Acción para el Empleo de Personas con Discapacidad, 2008-20I2". 
Referencias bibliográficas

Alcaide C. (2005): "La desigualdad en el mercado de trabajo de personas con discapacidad". Revista Universitaria de Ciencias del Trabajo, 6: I $47-164$.

Alomar, E. y Cabré, M. (2005): "El trabajo de jóvenes con discapacidad intelectual en entornos normalizados". Revista de Síndrome de Down: Revista Española de Investigación e Información sobre Síndrome de Down, 87: II 8-I 24 .

Barea, J. y Monzón, J. L. (2008): Economía social e inserción laboral de las personas con discapacidad en el País Vasco (en línea). <http:// www.fbbva.es/TLFU/dat/economia_social_ baja_pv_web.pdf $>$, acceso 7 de diciembre de 2016.

Boardman J. et al. (2003): "Work and employment for people with psychiatric disabilities". The British Journal of Psychiatry, I 82 (6): 467-468.

Calderón, M. J. y Calderón, B. (2012): “Los Centros Especiales de Empleo como mecanismo de tránsito hacia el mercado de trabajo ordinario". CIRIEC - España. Revista de Economía Pública, Social y Cooperativa, 75: 223-250.

Calvo, J. (2004): “Centros Especiales de Empleo: situación jurídica actual y perspectivas de futuro". Revista vasca de economía social, o: I39-I60.

Camacho-Miñano, M. M. y Pérez, R. (20I2): "Centros Especiales de Empleo: Empresas para una sociedad comprometida responsablemente". Responsabilidad Social Empresarial, I2: 77-90.

Carmona, P. et al. (2013): "Diagnóstico económico-financiero de la empresa cooperativa. Un estudio comparado de los años 2004 y 2007". REVESCO. Revista de Estudios Cooperativos, IIO: 43-95.

Cimera, R. E. et al. (2012): "Do sheltered workshops enhance employment outcomes for adults with autism spectrum disorder?". Autism, I6 (I): 87-94.
Cueto, B. et al. (2008): Trayectorias laborales de las personas con discapacidad y centros especiales de empleo: Análisis empírico con la muestra continua de vidas laborales (en línea). <http://www.seg-social.es/prdioo/groups/public/ documents/binario/I I 9779.pdf>, acceso $7 \mathrm{de}$ diciembre de 2016 .

De Andrés, J. (2000): “Caracterización económicofinanciera de las empresas asturianas en función de su nivel de rentabilidad”. RAE: Revista Asturiana de Economía, (I 8): I9I-222.

De Lorenzo, R. (2004): "El futuro de los discapacitados en el mundo: el empleo como factor determinante para la inclusión". Revista de ministerio del trabajo y asuntos sociales, 50 : 73-89.

España. Real Decreto Legislativo I/2013, de 29 de noviembre por el que se aprueba el Texto Refundido de la Ley General de derechos de las personas con discapacidad y de su inclusión social, Boletín Oficial del Estado, 3 de diciembre de 20I3, núm. 289, pp. $95635^{-}$ 95673 .

España. Real Decreto 469/2006, de 2 I de abril, por el que se regulan las unidades de apoyo a la actividad profesional en el marco de los servicios e ajuste personal y servicios de los Centros Especiales de Empleo, Boletín Oficial del Estado, 22 de abril de 2006, núm. 96, pp. I 5592 -I 5596.

España. Real Decreto 364/2005, de 8 abril de 2005 , sobre la regulación del cumplimiento alternativo con carácter excepcional de la cuota de reserva a favor de trabajadores con discapacidad, Boletín Oficial del Estado, 20 de abril de 2005 , núm. 94, pp. I3466I3469.

España. Real Decreto 290/2004, de 20 de febrero, sobre la regulación de los enclaves laborales como medidas de fomento de empleo de las personas con discapacidad, Boletín Oficial del Estado, 2 I de febrero de 2004, núm. 45, pp. 8386-839I. 
España. Real Decreto 27/2000, de I4 de enero, por el que se establecen medidas alternativas de carácter excepcional al cumplimiento de la cuota de reserva del 2 por Ioo en favor de trabajadores discapacitados en empresas de 50 o más trabajadores, Boletín Oficial del Estado, 26 de enero de 2000, núm. 22, pp. 34 I0-34I 2.

España. Real Decreto 2273/I985, de 4 de diciembre, por el que se aprueba el Reglamento de los Centros Especiales de Empleo definidos en el artículo 42 de la Ley I3/I982, de 7 de abril, de integración social del minusválido, Boletín Oficial del Estado, 9 de diciembre de I985, núm. 294, pp. 388 I I-388 I 2.

España. Ley I3/I982, de 7 de abril, de Integración Social de las personas con discapacidad, Boletín Oficial del Estado, 30 de abril de I982, núm. IO3, pp. I IIO6-IIII2.

Fernández, A. I., y García, M. (I99I): “Análisis del comportamiento económico-financiero de los sectores empresariales en España” (en línea). <http://repositorio.unican. es/xmlui/bitstream/handle/ı0902/3607/ Fern \% $\mathrm{C}_{3} \%$ Arndez \% 2c\% 20A.I.\% 2c\% \% Garc\% ${ }_{3} \% A D a \% 2 c \% 20 M . \% 2 c \% 20 I 99$ I. pdf? sequence $=\mathrm{I} \&$ isAllowed $=\mathrm{y}>$, acceso $7 \mathrm{de}$ diciembre de 2016 .

Fernández, E. et al. (I996): "Caracterización económico-financiera de la gran empresa industrial española según su rentabilidad". Revista Española de Financiación y Contabilidad: 343-359.

Gelashvili, V. et al. (20I 5): "Patrones de supervivencia para la gestión de los centros especiales de empleo". Revista de Estudios Empresariales. Segunda Época, I: I09-I26.

Giménez, S. (2012): Sinergias en tiempos de crisis: los centros especiales de empleo. Quaderns per a la Inclusió Social, 2, Tarragona: Publicaciones URV.

Gómez, J. M. et al. (2010): "Las dimensiones de la calidad del empleo en la economía social: un análisis de las Sociedades Laborales y de los Centros Especiales de Empleo en Castilla y León”. CIRIEC-España, Revista de Economía Pública, Social y Cooperativa, 67: 45-74.
Hernández, J. y Millán, J. M. (20I 5): “Las personas con discapacidad en España: inserción laboral y crisis económica”. Revista Española de Discapacidad (REDIS), 3 (I): 29-56.

Howard, M. (2005): "Disability and work". Public policy research, I2 (3): I $83-\mathrm{I} 87$.

Huete, A. y Díaz Velázquez, E. (20 I I): "Personas con discapacidad afectadas por el sistema penal penitenciario en España" (en línea). <http:// riberdis.cedd.net/bitstream/handle/I I I 8 I/2880/ personas_discapacidad_afectadas_sistema_ penal_penitenciario.pdf? sequence $=\mathrm{I} \&$ rd=003 I I $70332847944>$, acceso 7 de diciembre de 2016 .

Jordán de Urríes, F. B. y Verdugo, M. A. (20IO): "Informe sobre la situación de los Centros Especiales de Empleo en España” (en línea). $<$ http://sid.usal.es/idocs/F8/FDO25 I 53 / InformeMTIN_Centros_EspecialesdeEmpleo. pdf $>$, acceso 7 de diciembre de 2016.

KPMG (2013): Presente y futuro de los Centros Especiales de Empleo (en línea). <http://www. feaps.org/files/Documentos/CEEcompleto.pdf>, acceso 7 de diciembre de 2016 .

Laloma, M. (2007): Empleo protegido en España: Análisis de la normativa legal y logros alcanzados, Madrid: Grupo editorial Cinca, S. A.

Ministerio de Sanidad, Política Social e Igualdad (20I I): Estrategia Española sobre discapacidad 20I2-2020 (en línea). <https://www.msssi.gob. es/ssi/discapacidad/docs/estrategia_espanola_ discapacidad_20I 2_2020.pdf>, acceso 7 de diciembre de 2016 .

Ministerio de Trabajo e Inmigración (2008): Estrategia global de acción para el empleo de las personas con discapacidad. 2008-20I 2 (en línea). <http://www.empleo.gob.es/es/ sec_trabajo/Estrategia_global_personas_ discapacidad_2008_20I2.pdf $>$, acceso 7 de diciembre de 2016 .

Redondo, M. N. (2013): Comportamiento económico-financiero de los Centros Especiales de Empleo en Castilla y León. Años 200420 Io (Tesis doctoral). Facultad de Ciencias Económicas y Empresariales, Universidad de Valladolid. 
Redondo, M. N. y Martin, I. (2OI4): "Supervivencia de los Centros Especiales de Empleo en la Comunidad Autónoma de Castilla y León”. Revista de la Asociación Española de Contabilidad y Administración de Empresas, Io8: 25-28.

Rodríguez, G. et al. (2009): Evaluación de las políticas de empleo para personas con discapacidad y formulación y coste económico de nuevas propuestas de integración laboral, Madrid: Grupo editorial Cinca, S. A.
Rodríguez, V. (2OI2): "El empleo de las personas con discapacidad en la gran recesión: ¿Son los Centros Especiales de Empleo una excepción?”. Estudios de Economía Aplicada, I (30): 10-24.

Shepherd, G. (1989): "The value of work in the I980s." Psychiatric Bulletin, I3 (5): 23 I-233.

Skedinger, P. y Widerstedt, B. (2003): Recruitment to sheltered employment: Evidence from Samball, a Swedish state-owned company (en línea). <https://www.econstor.eu/ bitstream/IO4I9/822I2/I/Wpo3-II.pdf>, acceso 7 de diciembre de 2016 . 